\title{
Universal screening for sars-cov-2 in pregnant entered in an obstetrics hospitalization unit
}

\author{
Abstract \\ Objective: Determine the strengths and weakness of asymptomatic screening for COVID-19 \\ in pregnant women. Analyze the results an percentage of positives in asyntomatic pregnant \\ women. \\ Design: Descriptive retrospective observational study. \\ Setting: Mancha-Centro Hospital (Spain). \\ Population: Asymptomatic pregnant women that get in to the hospital. \\ Methods: Between the $12^{\text {th }}$ of April and $1^{\text {th }}$ of June 2020, all the asymptomatic pregnancies \\ were screened with diagnostic test for SARS-CoV-2. Data collection was done by reviewing \\ the medical records. Main outcomes measures: Clinical characteristics, management, \\ treatment, and obstetric and neonatal outcomes.
}

Results: A total of 117 patients were routinely screened using serological tests in materna blood and PCR in nasopharyngeal exudate to detect COVID-19 patients. Of the 117 patients screened, $24(20.5 \%)$ were positive in at least one of the two tests used for screening, of which $19(79 \%)$ were asymptomatic and $5(21 \%)$ symptomatic.

Conclusions: We recommend universal screening of all pregnant woman for COVID-19 during the pandemic because of the limits of the symptomatic screening seen in the actual literature and the importance of transmission between asymptomatics patients.
Volume 7 Issue 2 - 2021

\author{
Maria Pola Guillén, Elías Ortiz Molina, \\ Fiamma García Sánchez,Valentín Rodrigo \\ Álvaro, Elizabeth González Prieto,Ana \\ Pascual Pedreño \\ Department of Obstetrics and Gynecology, Hospital General La \\ Mancha Centro, Spain
}

Correspondence: María Pola Guillén, Department of Obstetrics and Gynecology Hospital General La Mancha Centro,Alcázar de San Juan, Avenida de la Constitución s/n Alcázar de San Juan 13600 ( Ciudad Real), Spain, Tel (+34) 667477865, Email Mariapola@hotmail.es

Received: February 25, 2021 | Published: April 28, 202 |

Keywords: screening in SARS-COV-2, pregnancy COVID infection

\section{Introduction}

The COVID 19 pandemic that is being suffered worldwide still has many unknowns, including the number of asymptomatic carriers of the virus that have been described as one of the major transmitters of the disease. It is estimated that between 72 to $90 \%{ }^{1}$ of those infected have not been diagnosed. Spain is the second country in number of infections with around 223,000. Our center, Hospital Mancha Centro, is one of the fourteen placed in a region of Spain called Castilla La Mancha. This area is one of the most affected areas of the pandemic in Spain with a mortality ratio of 174 deaths per 100,000 population, representing 11 of each 100 deaths of COVID-19 in Spain, numbers similar in the most devastated areas worldwide.

Once it has been known that asymptomatic carriers are potentially transmitters of the disease, it has been considered to carry out diagnostic tests on any patient admitted to a hospitalization unit or even to carry out massive tests on the population. In our hospital, routine screening has been established prior to the admission of any obstetric patient regardless of the reason for admission. Rapid serological testing and PCR of the SARS-CoV-2 is performed on all the patients admitted to our unit.

This measure was adopted in April. Asymptomatic carriers are the main source of infection for healthcare personnel, in addition to the limited availability of PPE reserved for suspected or symptomatic cases and / or with confirmation of infection.

\section{Methods}

\section{Study design and population}

This article presents a descriptive retrospective observational study on a non-probabilistic sample of asymptomatic pregnant women for
COVID -19 that get in to the Hospital because of obstetric pathology between the $12^{\text {th }}$ of April and the $1^{\text {th }}$ of June 2020.

The inclusion criteria were all pregnancies that are admitted to the hospital because of obstetrics pathology. All pregnant women admitted to our unit underwent a diagnostic screening for SARSCOVID using serological tests and nasopharyngeal PCR. There were no exclusion criteria.

\section{Statistical analysis}

Continuous variables were expressed as means with their standard deviation (SD). For the quantitative variables we defined their absolute and relative frequencies. All the statistical analysis is descriptive and has been carried out using the statistical software SPSS v24.0. No funding supports this study.

\section{Results}

A total of 117 patients were routinely screened using serological tests in maternal blood and PCR in nasopharyngeal exudate to detect COVID-19 patients. Of the 117 patients screened, 24 (20.5\%) were positive in at least one of the two tests used for screening, of which 19 (79\%) were asymptomatic and $5(21 \%)$ symptomatic (Table 1). The 7 pregnant women who initially reported symptoms had cough $(57.1 \%)$ as the most frequent symptom, followed by fever, anosmia and dyspnea. Subsequently, COVID-19 disease was ruled out in 2 cases (Table 1). Accuracy of maternal symptoms in predicting COVID-19 infection. The mean age of our analyzed sample was $31.3 \pm 5.8$ years, with the age range being between 13 and 43 years. Of the total of women, 108 pregnant women $(92.3 \%)$ were of Caucasian origin, 6 (5.1\%) from North Africa and 3 (2.6\%) were American. The mean gestational age was 36 weeks. Among the asymptomatic patients, we 
obtained a positive serological test result in $15.5 \%$ (17 patients) and $5.5 \%$ of positive PCR results (6 patients), thus concluding that 11 patients would have passed the disease at the time of screening.

Table I Accuracy of maternal symptoms in predicting COVID-I9 infection

\begin{tabular}{llll}
\hline & $\begin{array}{l}\text { Positive } \\
\text { COVID-19 }\end{array}$ & $\begin{array}{l}\text { Negative } \\
\text { COVID-19 }\end{array}$ & TOTAL \\
\hline Symptomatic & 5 & 2 & 7 \\
Asymptomatic & 19 & 91 & 110 \\
Total & 24 & 93 & 117
\end{tabular}

When asked about close contact with people who could have transmitted the disease, only $22.7 \%$ of the asymptomatic group reported having had any health contact or with sick relatives. Regarding the type of delivery, an increase in the percentage of cesarean section is observed in positive cases compared to negative cases, although this difference is not statistically significant (Table 2). Results; type of delivery according to the test result. Twelve infants of positive COVID-19 mothers have been tested and all of them have had a negative result. ${ }^{2}$

Table 2 Results: type of delivery according to the test result

\begin{tabular}{llllll}
\hline & & Eutocic & Instrumental & Cesarean & $\mathbf{p}$ \\
\hline & & $(\mathbf{n}=\mathbf{6 8})$ & $\mathbf{( n = 1 3 )}$ & $\mathbf{( n = 2 3 )}$ & \\
\hline $\begin{array}{l}\text { Results } \\
\text { Global }\end{array}$ & Negative & $55(64.7 \%)$ & $12(14.1 \%)$ & $18(21.2 \%)$ & $0.55 \mid$ \\
Test & Positive & $13(68.4 \%)$ & $\mid(5.3 \%)$ & $5(26.3 \%)$ & \\
\hline
\end{tabular}

\section{Discussion}

The need for implementation of a universal screening for all the pregnant women admitted to the labour room has been one of the main discussions of the management. In those hospitals where it has been done, the prevalence of COVID-19 in asymptomatic patients published is $13.5 \%{ }^{3}$ and $13 \% .{ }^{4}$ In our setting, the incidence of the disease has been one of the highest at the level of our country, so we have been subjected to a constant challenge regarding the diagnosis, management and treatment of the patients who entered our department.

Our results offer a slightly increased percentage with respect to those observed in previous studies, reaching $20.5 \%$ of positive pregnant women in the asymptomatic group. After reviewing the literature, the development of the different diagnostic methods of this new pathology is particularly important, as well as the management of patients in the hospital setting. The organization and separation of patients who can transmit the disease at both an extra and in-hospital level in order to control the spread of the virus is currently a great challenge for the health system: hence the screening and evaluation methods for this new pathogen are especially important today.
A lot of unknowns continue to exist regarding the management of asymptomatic patients as well as the criteria to carry out an adequate screening. In our center we have chosen to perform serological tests and nasopharyngeal PCR on all obstetric patients at the time of admission. It is worth noting the high percentage of asymptomatic patients with whom we have found what makes it even more relevant to carry out an adequate screening of the patients.

\section{Details of ethics approval}

Local IRB (Institutional Review Board) was consulted, being this article exempt from the need for approval because of the noninterventional, observational study and the sanitary emergency. This work has also the approval of the medical director of our center and each pregnant woman was informed about the study and gave their consent to use their clinical information. The ethics principles have been respected. All authors agree to be accountable for all aspects of the work in ensuring that questions related to the accuracy or integrity of any part of the work are appropriately investigated and resolved

\section{Acknowledgments}

None.

\section{Contribution to authorship}

MPG and EOM conceived the study question. FGS,VRA, EGP, collected the data. FGS, VRA performed the statistical analyses. EOM, MPG and FGS drafted the first version of the manuscript and it was revised and approved by APP.

\section{Conflicts of interest}

All the authors declare no competing interests. Completed disclosure of interest forms are available to view online as supporting information.

\section{Funding}

None.

\section{References}

1. Li R, Pei S, Chen B, et al. Substantial undocumented infection facilitates the rapid dissemination of novel coronavirus (SARS-CoV2). Science. 2020 .

2. William S Vintzileos, Jolene Muscat, Eva Hoffmann, et al. Screening all pregnant women admitted to labor and delivery for the virus responsible for coronavirus disease 2019. AJOG. 2020;223(2):284-286.

3. Sutton D, Fuchs K, D'Alton M, et al. Universal Screening for SARSCoV-2. Women Admitted for Delivery. $N$ Engl J Med. 2020.

4. Vintzileos WS, Muscat J, Hoffmann E, et al. Screening all pregnant women admitted to Labor and Delivery for the virus responsible for 1 COVID-19 2. Am J Obstet Gynecl. 2020;S0002-9378(20)30472-5. 